\title{
Security Attacks in Mobile Ad hoc Networks (MANET): A Literature Survey
}

\author{
Dinesh \\ Assistant Prof. \\ Sri Sai lqbal Collge of \\ Management \& IT, \\ Badhani, Punjab, India
}

\author{
Ajay Kumar \\ Associate Prof. \\ ECE Deptt,BCET \\ Gurdaspur, Punjab, India
}

\author{
Jatinder Singh \\ Principal \& Prof. \\ KC College of Engg. \& IT \\ Nawanshar, Punjab, India
}

\begin{abstract}
Security is actually an essential issue for the secured conversation in between mobile nodes in a dangerous ecosystem. In dangerous situations adversaries can easily group active and inactive assaults opposing intercept able routing in implant inside routing information as well as data packets. In this specific document, we have pay attention to essential security symptoms within Mobile adhoc networks. MANET has no definite defensive structure, so that, it is easily accessible to both trustworthy networking end-users as well as destructive assailants. Within the existence of malware nodes, one of the primary obstacles about MANET is always to develop the sturdy safety alternative which is able to shield MANET from a variety of routing attacks. Conversely, these types of alternative are certainly not appropriate MANET resource limitations, in other words, restricted bandwidth and electric battery power, simply because they introduce hefty traffic bunch to exchange and validating important factors. MANET can run in reclusiveness or perhaps in dexterity by having a wired infrastructure, frequently through the gateway node actively participating both in networks for traffic relay. This Particular versatility, together with their self organizing potential, are a few of MANET's most significant strengths, in addition to their leading safety weak points. In this particular review paper a variety of routing assaults, such as for instance active(flooding, black hole, spoofing, wormhole) and inactive(eavesdropping, traffic monitoring, traffic analysis) are characterized.
\end{abstract}

\section{Keywords}

MANET, Mobile Ad hoc Network, Security Attacks in MANET, Denial of Service attack

\section{INTRODUCTION}

In ${ }^{[12,13,14]}$ Mobile Ad Hoc Networks (MANETs) has recently turned out to be the most predominant aspects of research in the present-time simply because for the issues that it present towards the associated methodologies.

MANET certainly is the new growing technologies which makes it possible for end-users to communicate without worrying about any physical infrastructure irrespective to their particular geographical location, that's why it is occasionally labeled as being an "infrastructure less" network. The expansion of inexpensive, smaller than average more substantial devices transforms MANET a fastest growing network. An adhoc network is self coordinating as well as transformative. Appliance in mobile ad hoc network should really have the ability to identify the existence of various other devices and execute essential set up to improve interaction as well as sharing of information and service ${ }^{[1]}$. Ad hoc networking makes it possible for the devices to maintain connections to the network as well as effortlessly incorporating and eliminating devices to and from the network. The set of programs for MANETs is assorted, which range from large-scale, mobile, extremely vibrant networks, to small, static networks that are restricted by power resources. Aside from the legacy applications that relocate from conventional infrastructure environment into the ad hoc framework, a excellent deal of brand new services and will be developed for the new ecosystem. It includes:
1. Armed Forces Battlefield ${ }^{[14]}$
2. Sensing Unit Systems
3. Healthcare Service
4. Personal Area Network.

Security possibilities are essential concerns for MANET, especially for those finding hypersensitive programs, have to satisfy the following design objectives although dealing with the above mentioned challenges. MANET is more susceptible compared to wired network because of to mobile nodes, hazards coming from affected nodes within the network, restricted physical security, compelling topology, scalability as well as shortage of centralized administration. As a result of these types of vulnerabilities ${ }^{[7]}$ MANET tend to be more susceptible to destructive assaults. The principle focus of this particular tasks are to present a study upon a lot of different attacks that influence the MANET behavior as a result to virtually any explanation.

\section{LITERATURE REVIEW}

A MANET is a most appealing and also growing rapidly innovation that is based upon a self-organized and swiftly implemented network. As A Result for their amazing features, MANET appeals to separate real-world application segments where in actuality the networks topology variations extremely at a fast rate. The present safeguards possibilities of wired networks cannot be practiced straight away to MANET, which in turn is really a MANET allot more susceptible to security assaults. In this document, we certainly have talked about existing routing assaults inside MANET. Proactive studies work with MANETs is actually carrying-on primarily about the fields of Medium Access Control (MAC) ${ }^{[4,18]}$, routing, useful resource administration, electric power control, and safety. Simply Because for the significance of routing standards inside vibrant multihop ${ }^{[8]}$ networks, a lot of MANET routing standards have already been recommended within the last couple of years. Along with the specialized characteristics of MANET, whenever thinking of any routing method, typically the subsequent characteristics tend to be expected, although each one of these won't be achievable to include inside a solitary alternative.

- A routing communications protocol for the MANET should always be dispersed within form in order to really boost their trustworthiness ${ }^{[15,18]}$ 
- A routing protocol need to become tailored contemplating unidirectional connections mainly because wireless media could potentially cause a wireless associate to become exposed in unidirectional just because actual physical aspects.

- The routing protocol ought to be power streamlined.

- The routing protocol ought to give consideration to their protection.

- A hybrid routing method ought to be significantly more activated compared to enthusiastic in order to prevent operating expense.

- A routing protocol should really be familiar with excellent Service

Ad hoc networking is not a new concept. As a technology for dynamic wireless networks, it has been deployed in military since 1970s. Commercial interest in such networks has recently grown due to the advances in wireless communications. A new working group for MANET has been formed within the Internet Engineering Task Force (IETF), aiming to investigate and develop candidate standard Internet routing support for mobile, wireless IP autonomous segments and develop a framework for running IP based protocols in ad hoc network In the trailing section, existing routing problems as well as its countermeasures in opposition to MANET standards tend to be reviewed thoroughly ${ }^{[16]}$

\section{MANET AMENABLENESS}

A susceptibility is actually a weak spot inside security measures. A specific system might be susceptible in order to unauthorized information control considering that the system really does not validate a user's identification prior to permitting information accessibility. MANET is much more susceptible compared to wired network. A Few associated with the weaknesses tend to be as follows:

\subsection{Scalability}

Because Of portability of nodes, degree of scale for adhoc network varying almost all the time period. So that scalability is actually a significant problem with regards to security. Security method should always be proficient associated with maneuvering a significant network as well as tiny ones.

\subsection{Cooperativeness}

Routing algorithmic rule for the MANETs generally believe that nodes tend to be accommodative as well as nonmalicious. Due to this fact a malware assailant can very quickly turned out to be a significant routing representative and disrupt network functioning simply by infringe the protocol specific features.

\subsection{Useful Resource availableness}

Powerful Resource accessibility is actually a significant problem in MANET. Promoting protected communications in this kind of changing ecosystem and protective covering in opposition to certain hazards as well as destruction, contributes to growth of assorted security strategies as well as architectures. Collective ad-hoc environments always permit utilization of self-organized security method.

\subsection{Deficiency of central administration}

Deficiency of central administration: MANET does not possess a focused monitor hosting server. The lack associated with administration may seem to make the recognition of attacks complicated because it's not really ease to monitor the network traffic in a extremely dynamic and large degree ad- hoc network. Deficiency of centralized administration will certainly hinder reliability management for the nodes

\subsection{Bandwidth Restriction}

Adjustable minimal capacity links is available as contrasted to wireless network which in turn tend to be more vulnerable to additional disturbances, interference and signaling attenuation consequence.

\subsection{No predetermined perimeter:}

Within mobile ad- hoc networks we are unable to exactly determine an actual perimeter for the system. The nodes work as part of the nomadic ecosystem where exactly they're permitted to become a member of and then leave the wireless network. The moment an adversary appear within the broadcast range of a node it will likely have the ability to communicate with just that node. The symptoms consist of Eavesdropping impersonation; tempering, replay and Denial of Service (DoS) attack

\subsection{Constrained Power Resources}

The nodes inside mobile ad-hoc network really want to take into consideration limited power-supply, which will undoubtedly influence countless hassles. A node in mobile adhoc network may perhaps conduct themselves in a egotistic way when it's discovering that there exists exclusively limited ${ }^{[2]}$.power supply

\subsection{Resistance within the Network}

The mobile nodes inside the MANET can easily openly join and go out of the network. The nodes within network may perhaps usually react maliciously. This might be difficult to identify that the tendencies associated with the node is actually destructive ${ }^{[3]}$. Therefore this particular assault is much more harmful as compared to exterior attack. These Types Of nodes are known as affected nodes

\section{AREA OF APPLICATIONS FOR MANET}

With the enhancement in handheld equipment as well as advancement in wireless telecommunications, ad-hoc networking is gaining significance with all the growing quantity of extensive programs. Ad-hoc networking can be utilized everywhere whenever there can be minimal communication infrastructure or the existing infrastructure is costly or perhaps awkward to make use of. Ad hoc networking enables the equipment to help keep connectivity towards network as well as conveniently incorporating as well as eliminating devices to and from the network. The set of programs for the MANET is actually diverse, which range from extensive, mobile phone, extremely strong networks, to small, fixed networks that are restricted through electrical power sources. In Addition To their legacy programs which wiggle from conventional infra structured environment into the ad hoc framework, a large amount of brand new services can and will be developed for all the better environment.

\subsection{Military Battlefield}

Armed forces devices nowadays consistently consists of some kind of computer system equipment. Ad- hoc networking would definitely enable the armed forces to consider benefit from very common network technology to uphold an important information network between the troops, vehicles, as well as armed forces information headquarters. The fundamental strategies of ad hoc network emerged because of this discipline. 


\subsection{Personal Area Network (PAN):}

\section{Short range MANET can easily streamline the} intercommunication in between a variety of mobile devices (such as a PDA, a notebook, as well as a mobile phone). Monotonous wired cables are swapped out alongside wireless links. This type of ad hoc network can additionally extend the availability to the web or any other networks by mechanisms e.g. Wireless LAN (WLAN), GPRS, and UMTS. The PAN is probably a encouraging application field of MANET in the foreseeable future pervading processing framework.

\subsection{Commercial Sector:}

Ad hoc can easily be utilized in unexpected emergency recovery operations for disaster alleviation attempts, for example. In open fire, flood, or earthquake. Unexpected Emergency recovery procedure must simply take place exactly where non-existing or possibly wrecked communications infrastructure and accelerated implementation of a communication network is recommended. Data is relayed from a single rescue team member to the other compared to a small hand held. Some Other commercial scenarios incorporate for example. ship-toship ad hoc mobile communication, law administration, and so on.

\subsection{Local Level:}

Ad hoc networks can autonomously connect an instant as well as momentary multimedia system network using notebook, Laptop or palmtop computers to dispersed as well as promote information amongst respondents at for example. meeting or perhaps workshop. Another recommended localized standard application might be in home networks exactly where equipments can communicate immediately in order to exchange important information. In a similar fashion various other civilian environments like taxicab, sports stadium, motorboat as well as compact craft, cell phone ad hoc communications will have countless programs.

\section{ATTACKS IN MANET}

Protecting wireless ad-hoc computer networks is a extremely challenging subject. Learning about feasible type of problems is almost always the initial step in direction of establishing effective safety alternatives. Security of communications within MANET is extremely important for the secured transmission of data.Absence of any other fundamental co ordination procedure as well as distributed wireless medium tends to make MANET a lot more susceptible to digital/cyber assaults compared to wired network generally there are really a wide range of attacks that influence MANET ${ }^{[15]}$. These types of assaults is generally categorized in to two types:

1.Internal Attack: Inner assaults come from affected nodes which are a segment associated with the network. In an inner attack the malicious node from the network benefits unauthorized connection and also impersonates being a authentic node. It would possibly examine targeted traffic in between some other nodes and may also get involved in another network activities

2.External Assault: External attacks tend to be performed by nodes which do not are members of the network. It triggers obstruction sends fake routing facts or perhaps results in inaccessibility to services.

\subsection{Denial of Service attack:}

In a denial-of-service (DoS) attack, an assailant tries to restrict trustworthy end-users from being able to access important information or perhaps services. By concentrating on your computer or laptop as well as its network connections, or even the computer systems as well as network of the sites you are attempting to make use of, an assailant might possibly keep you from being able to access e-mail, web sites, online profile (banking, etc.), or some other services that be dependent regarding the influenced computer.

Almost each typical as well as apparent kind of DoS attack happens whenever an attacker "surges" a network alongside information. Whenever you enter a website url with regard to specific internet site into the web browser, you are actually forwarding a request to that site's computer hosting server to review the page. The hosting server is only able to processes a specific amount of data requests at a time, therefore if an attacker overloads their hosting server with requests, it can't process your demand. This can be a "denial of service" because you are unable to access that site.

An assailant can make use of junk e-mail messages to launching an identical assault on your own email account. Regardless Of Whether you've got an e-mail accounts offered by your employer or one conveniently readily available throughout the complimentary services such as for instance Yahoo or Hotmail, you are allocated a particular allotment, which in turn restrictions the actual quantity of information you could have within your accounts at any moment. By sending numerous, or perhaps spacious, email messages to the account, an assailant can easily ingest your own allotment, protecting against through acquiring trustworthy emails.

This assault is designed to attack the availableness associated with a node or perhaps the whole network. If the attack is worthwhile the support may not be available to you

\subsection{Black Hole Attack}

This dangerous node advertises its availableness about refreshed channels regardless of verifying it's routing table. In this particular approach assailant node will usually experience the accessibility in responding to the route ask for and therefore intercept the information packet and preserve it . In protocol formulated upon flooding, ${ }^{[20]}$ the malware node response will likely be accepted through the requesting node just before the reception of respond back from genuine node; consequently a malicious and forged route is planned. The moment this path is establish, now it's up to the node regardless of whether to decrease all of the packets or forwards it to the unidentified target.

\subsection{Wormhole Attack}

Wormhole attack is a sort of replay attack that is especially frustrating in MANET to shield against. Even if, the routing facts is sensitive, encoded or perhaps authenticated, it could be extremely effective furthermore detrimental. An assailant can easily tunnel a request packet RREQ immediately towards desired destination node without worrying about enhancing the hop-count benefits. Therefore it inhibits every other paths from being determined. It might probably badly interrupt communications as $\mathrm{AODV}^{[7,9]}$ would definitely not be able discover paths more than a small number of hops. It is effortless when it comes to assailant to really make the tunneled packet appear with much better metrical compared to a typical multi-hop path for the tunneled rides and distances longer than the regular transmitting selection of a single hop. Malware nodes can retransmit eavesdropped information once again inside a route this is certainly precisely accessible to attacker. The wormhole attack can be combined with all the information shedding attack to protect against the desired 
destination node from acquiring packets. wormholes tend to be harmful because they could undertake damage without worrying about even determining the network

\subsection{Rushing Attack}

First of all the lexicon concept of 'RUSHING ATTACK' is "a sudden attack", or "a sudden forward motion", or, "to execute, complete, or perhaps conclude with accelerate, impetuosity, or perhaps violence". In networking "RUSHING ATTACK" is also known as as "novel attack" or "denial of service" attack ${ }^{[5]}$. The rushing attack is a harmful attack $\mathrm{w}$

hich will act as a highly effective self-denial of service attack against all presently recommended on-demand ad hoc network routing protocols ( e.g. DSR , AODV etc), including protocols that were crafted to-be protected (e.g. Ariadane, ARAN etc) ${ }^{[4]}$. As we understand in on demand routing protocol, the path finding is really a process through which a source node acquires a route to desired destination node whenever would like to send out a packet to it .Normally, the source node receives an appropriate source route by searching its route cache of routes previously discovered ,but if no path can be found in their memory cache, it will probably start the path finding through flooding the path request $\left(\operatorname{RREQ}\left[{ }^{16]}\right)\right.$ to the network. To restrict the operating expense for this overflow, almost every node typically forwards exclusively one route request (RREQ) coming from any other path finding. If a accelerated transmitting route (for example. a devoted route distributed simply by assailants) exists between the two ends of the wormhole, the tunneled packets can travel more rapidly compared to those through the typical multi-hop path. The running attack can act as an effective denial-ofservice attack in opposition to almost all generally recommended on-demand MANET routing protocols, including protocols that were planned to be secured, for example ARAN and Ariadne[22]

\subsection{Flooding}

Malware nodes might also provide bogus packets into the network, or create ghost packets which trap across as a result of bogus routing facts, effectively using up the bandwidth and processing resources alongside the way in which. It has specifically dedicated consequence upon ad hoc networks, since the nodes of these types of generally possess only restricted assets with respect to electric battery as well as computational power ${ }^{[21]}$. Traffic may also be a financial aspect, according to the services available, so that whatever flooding which strikes within the website traffic statistics of the network or a specific node may result in substantial damage cost

\subsection{Jamming Attack}

It is a type of DOS challenge. There are plenty of assault techniques that the jammer is capable of doing to be able to affect some other wireless communications. A Number Of feasible techniques are exposed below:

- Persistent Jammer: a consistent jammer constantly produces a broadcast signal that represents random bits; the signal generator does not understand whatever MAC protocol ${ }^{[22]}$.

- Deceitful Jammer: completely different from the prolonged jammers, deceitful jammers don't transmit arbitrary pieces as an alternative they transmit semi-valid packets. This indicates that the packet header is appropriate however the payload is worthless $^{[16]}$

- Ergodic Jammer: Alternates anywhere between slumbering as well as jamming their channel. In the very first setting the jammer jams ${ }^{[13]}$ for a arbitrary time frame (it would possibly react either like a persistent jammer or perhaps a deceitful jammer), as well as in the next mode (the sleeping mode) the jammer transforms their transmitters off for an additional arbitrary time frame ${ }^{[17]}$. The vitality efficiency is determined as the ratio of the length of the jamming period over the length of the sleeping period.

- Reactive Jammer ${ }^{[9]}$ : A reactive jammer tries not to waste resources by only jamming whenever it sensory faculties which someone is transferring. Their target is not the transmitter nevertheless the recipient, attempting to input as a great deal noise as possible in the packet to modify as countless bits as feasible considering that merely a minimum amount of power is required to customize enough bits so that when a checksum is carried out over that packet at the recipient it's going to be known as not really appropriate and as a consequence thrown away.

In jamming ${ }^{[6]}$, assailant originally remember to keep tracking wireless medium in order to figure out rate of recurrence from which desired destination node is acquiring transmission coming from sender. It then transmit signal on that rate of recurrence to ensure that error free receptor is restricted.

\section{SECURITY GOALS}

Protection requires a collection of assets which are sufficiently moneyed. In MANET, many of the networking capabilities such as for instance routing as well as packet forwarding, are performed by nodes independently within a self organizing way. Therefore, locking down a mobile ad hoc network is extremely complicated. The goals to analyze in case mobile adhoc network is safe or otherwise not tend to be as follows:

Availableness: Availableness means that the resources have always been available to certified activities at relevant occasions. Accessibility is applicable both of to data and to services. It guarantees that the survivability concerning network service regardless of denial of service attack ${ }^{[10]}$.

Privacy: Secrecy will help to make sure that computer related resources tend to be utilized exclusively by certified person. That is, exclusively those people that really should have accessibility a little something will in reality get that accessibility. In order to maintain privacy of individuals sensitive facts, we have to keep them mystery ${ }^{[11]}$ from all agencies which do not have exclusive right to gain access to them. Privacy ${ }^{[18]}$ is frequently known as secrecy or solitude.

Integrity: Ethics implies that resources tends to be altered exclusively by authorized people or perhaps merely as part of certified way. Alteration consists of crafting, switching state, erasing as well as producing. Ethics guarantees that a information currently being transmitted has never been corrupted. $^{[14,7]}$

Authentication: Verification ${ }^{[12]}$ makes it possible for a node to ensure the identity of equal node it is communicating with. Verification is primarily belief just that people in interaction are authenticated and not impersonators. Reliability is actually ascertained because only the trustworthy transmitter may establish a communication that will decrypt perfectly because of the shared key ${ }^{[19]}$.

Non repudiation: Non repudiation makes sure that transmitter as well as recipient associated with a message are incapable to disclaim that they have ever before transmitted to accepted 
this kind of message. This Particular is effective as soon as we really need to discriminating in cases where a node along with some undesirable function is sacrificed or maybe not.

Anonymity: Anonymity means that almost all important information which can be accustomed recognize proprietor or perhaps existing user of node ought to standard be retained exclusive rather than be dispensed through node by itself or even the system software.

Endorsement: This particular feature assigns a variety of access rights to countless forms of end-users. For instance a network management can easily be practiced by network supervisor only ${ }^{[20]}$.

\section{CONCLUSION}

In this document, we've examined the safety hazards an adhoc network confronts as well as introduced the safety unbiased that require to be accomplished. On a single hand,the security-sensitive programs of an ad-hoc networks need to get extreme level of safety on the other side ,adhoc network are inevitably susceptible to safety assaults. Subsequently, there exists a intend to make all of them safer as well as resilient in order to accommodate the strenuous specifications among these networks.

The foreseeable future concerning to ad- hoc networks is genuinely enticing, offering the experience of - at any time, everywhere as well as inexpensive communications. Earlier all those envisioned situations become a reality, large amount of work is going to be carried out in simultaneously research and execution. At current, the typical tendency within MANET is in the direction of meshing structures as well as large-scale. Enhancement as part of bandwidth and potential is actually required, which means the necessity for a larger regularity ,a lot spatial spectral reuse.

In all, even though the extensive implementation of ad- hoc systems continues to be year away, the investigation within discipline will certainly carry on staying extremely effective and also extremely creative

\section{ACKNOWLEDGEMENT}

I am Thankful To Punjab Tecnical University Jalandhar Which Gives Me Opportunity To Work As Research Scholar.

\section{REFERENCES}

[1] A Mishra and K.M Nadkarni, security in wireless Ad hoc network, in Book. The Hand book of $\mathrm{Ad}$ Hoc Wireless Networks (chapter 30), CRC press LLC, 2003

[2] Jie $\mathrm{Wu}$, Fei Dai, -Broadcasting in Ad Hoc Networks: Based on Self-Pruning\|, Twenty Second Annual Joint Conferences of IEEE Computer and Communication Societies, IEEE INFOCOM 2003

[3] K. Sanzgiri, B. Dahill, B.N. Levine, C. shield and E.M Belding- Royar, A secure routing protocol for Ad Hoc Networks, in Proceedings of ICNP'02,2002.

[4] Y. Hu, D. Johnson and A Perrig, SEAD: Secure Efficient Distance Vector Routing for Mobile Wire

[5] D. Johnson and D. Maltz, -Dynamic Source Routing in Ad Hoc Wireless Networks, $\|$ Mobile Computing, T. Imielinski and H. Korth, Ed., pp. 153-81. Kluwer, 1996.

[6] C.E.Perkins and P. Bhagwat, - Highly dynamic destination-sequenced distance vector routing for mobile computers\|, Comp, Comm. Rev., Oct.1994, pp 234-44
[7] M. Frodigh, P. Johansson, and P. Larsson.-Wireless ad hoc networking: the art of networking without a network,\| Ericsson Review,No.4, 2000, pp. 248-263.

[8] E. M. Royer and C-K Toh, -A review of Current routing protocols for Ad Hoc Mobile Wireless.

[9] Chlamtac, I., Conti, M., and Liu, J. J.-N. Mobile ad hoc networking: imperatives and challenges. Ad Hoc Networks, 1(1), 2003, pp. 13-6.

[10] HaoYang, Haiyun \& Fan Ye - Security in mobile adhoc networks : Challenges and solutions,\|, Pg. 38-47, Vol11, issue 1, Feb 2004.

[11] Luis Bernardo, Rodolfo Oliveira, Sérgio Gaspar, David Paulino and Paulo Pinto A Telephony Application for Manets: Voice over a MANET-Extended JXTA VirtualOverlay Network

[12] C.-C. Chiang, "Routing in Clustered Multihop, Mobile Wireless Networks with Fading Channel," Proc. /E€€ SICON '97,Apr. 1997, pp. 197-211

[13] Th. Clausen et al., "Optimized Link State Routing Protocol,"

IETF Internet draft, draft-ietfmanet-olsr-11.txt, July 2003.

[14] B. Kannhavong, H. Nakayama, Y. Nemoto, N. Kato, A. Jamalipour. A survey of routing attacks in mobile ad hoc networks. Security in wireless mobile ad hoc and sensor networks, October 2007, page, 85-91

[15] Z. Karakehayov, "Using REWARD to Detect Team BlackHole Attacks in Wireless Sensor Networks," Wksp. Real-World Wireless Sensor Networks, June 20-21, 2005.

[16] S. Desilva, and R. V. Boppana, "Mitigating Malicious Control Packet Floods in Ad Hoc Networks," Proc. IEEE Wireless Commun. and Networking Conf., New Orleans, LA,2005.

[17] S. Lee, B. Han, and M. Shin, "Robust Routing in Wireless AdHoc Networks," 2002 Int'l. Conf. Parallel Processing Vancouver, Canada, Aug. 18-21, 2002.

Wksps.,

[18] S. Kurosawa et al., "Detecting Blackhole Attack on AODVBased Mobile Ad Hoc Networks by Dynamic Learning Method,'Proc. Int'l. J. Network Sec., 2006.

[19] D. Johnson and D. Maltz, "Dynamic Source Routing in Ad Hoc Wireless Networks," Mobile Computing, T. Imielinski and H. Korth, Ed., pp. 153-81. Kluwer, 1996.

[20] Jyoti Raju and J.J. Garcia-Luna-Aceves, “ A comparison of On-Demand and Table-Driven Routing for Ad Hoc Wireless networks'," in Proceeding of IEEE ICC, June 2000.

[21] Y-C. Hu, A. Perrig, and D. Johnson, "Wormhole Attacks in Wireless Networks," IEEE JSAC, vol. 24, no. 2, Feb. 2006.

[22] C. Perkins and E Royer, "Ad Hoc On-Demand Distance Vector Routing," 2nd IEEE Wksp. Mobile Comp. Sys. And Apps., 1999. 